\title{
A Novel Image Segmentation Method in Forestry Detection
}

\author{
Zheng Yu, Lei Yan*, Xiaokang Ding, Jianlei Kong and Ning Han \\ School of Technology, Beijing Forestry University, Beijing, China \\ *Email: mark_yanlei@bjfu.edu.cn
}

\begin{abstract}
Information of trees is a vital component in forestry intelligent monitoring and identification. In this paper, a novel live wire segmentation algorithm based on PCNN and Hessian matrix was proposed to extract information of trees, and infrared thermal images which contained trees were collected in forests. With the help of the proposed algorithm, we could capture the precise information of trees, which was crucial for forestry monitoring and management.
\end{abstract}

Keywords: Forestry detection, Image segmentation, Live wire, PCNN, Hessian matrix

\section{Introduction}

Tree's edge is closely related to its growth degree, which is a vital data in forestry management and production. It works as guidance to get fellable target automatically for harvester, and provides the trees' information for human. Thus, it is crucial to obtain information about trees' contour precisely. However, because of the complicated environment in forests, it is difficult to capture the contour of target precisely in visible light image. Infrared thermal image technique with high contrast between targets and background has been made inroads in many domains. In Ref. [1], infrared thermal image is used to monitor clinically significant measurement. Kontos detected primary breast cancer with the help of infrared thermal images [2]. Zia obtained information about water stress from infrared thermal images which is significant association with yield and plant height [3]. And in Ref. [4], fused with visible image, infrared thermal image is used to detect forestry situation. Thus, infrared thermal image technique provides a feasible approach to capture the contour of trees.

Recently, the study on image segmentation algorithm has become a hot spot in medical image processing, agricultural management, and military monitoring. For example, coronary vessel was extracted in physic image by Miu [5]. Geng exactly captured interested objects in physic image with better segmentation precision and rate [6]. Thapaliya proposed a method for segmenting brain tumor images from MR images [7]. Sammouda monitored the actual bee forage areas in satellite image based on image segmentation method [8]. And Artur recognized lesion area in medical image with an improved image segmentation method [9].

In this paper, a PCNN and Hessian based live wire segmentation method was proposed and applied to detecting trees' information in forests.

\section{Image Segmentation Method}

Live wire segmentation method was firstly proposed by Barrett and Mortensen in 1992 [10], which is a widely used tool to obtain boundaries in images. As shown in figure 1, the procedure of this method can be divided into two parts. 


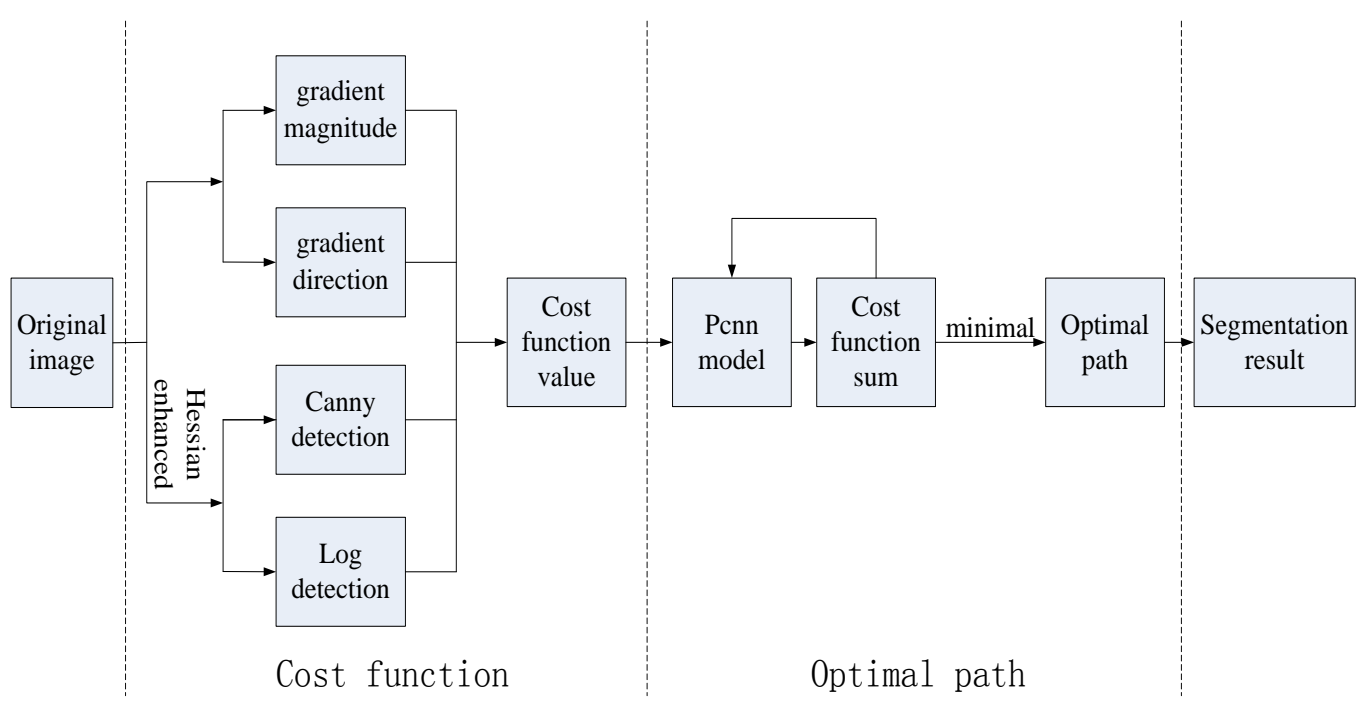

Figure 1. Procedure of Proposed Method

Firstly, the cost function at each pixel is defined based on the eight neighbor pixels, and then, an optimal path is determined through searching from start pixel to the goal pixel (which can be artificially marked by users) according to the cost function between neighbor pixels. During this process, PCNN is used for searching optimal path, and Hessian matrix is for enhancing the image so as to get a more accurate cost function.

\subsection{Live Wire Segmentation}

2.1.1 Cost Function: This method could capture objects boundaries in images from provided start node to the goal node. The cost function between the neighbor pixels $\mathrm{p}$ and $\mathrm{q}$ is defined as formula 1 :

$$
f(\mathrm{p}, \mathrm{q})=\omega_{\text {Log }} f_{\text {Log }}(\mathrm{q})+\omega_{\text {canny }} f_{\text {canny }}(\mathrm{q})+\omega_{G} f_{G}(\mathrm{q})+\omega_{D} f_{D}(\mathrm{p}, \mathrm{q})
$$

Where $\omega$ represents the weight of each feature, $f_{\text {Log }}(q)$ is the result of Log edge detection in pixel q, $f_{\text {canny }}(q)$ represents the canny edge detection, $f_{G}(q)$ refers to gradient magnitude, and $f_{D}(\mathrm{p}, q)$ refers to the gradient direction from pixel $\mathrm{p}$ to $\mathrm{q}$.

In the theory of live wire, the image can be regarded as a connection matrix with a cost function between neighbor pixels, and a low cost function means a strong edge. Thus, we set $f_{\text {Log }}(q)$ and $f_{\text {canny }}(q)$ as the adverse aspects of edge detection result. As for gradient magnitude and gradient direction, we take measures as follows [11]:

$$
f_{G}(\mathrm{q})=1-\frac{G}{\max (\mathrm{G})}
$$

Where $G=\sqrt{I_{x}^{2}+I_{y}^{2}}$, and $I_{x}, I_{y}$ are the gradient components in each direction.

$$
f_{D}(\mathrm{p}, \mathrm{q})=\frac{1}{\pi}\left\{\cos \left[\mathrm{d}_{p}(\mathrm{p}, \mathrm{q})\right]^{-1}+\cos \left[\mathrm{d}_{q}(\mathrm{p}, \mathrm{q})\right]^{-1}\right\}
$$

where $\quad d_{p}(\mathrm{p}, \mathrm{q})=\mathrm{D}^{\prime}(\mathrm{p}) \bullet L(\mathrm{p}, \mathrm{q}), \quad d_{q}(\mathrm{p}, \mathrm{q})=L(\mathrm{p}, \mathrm{q}) \bullet \mathrm{D}^{\prime}(\mathrm{q}) \quad$ and $\quad D^{\prime}(\mathrm{p})=\left(\mathrm{I}_{y}(\mathrm{p}),-\mathrm{I}_{x}(\mathrm{p})\right)$, in which $L(\mathrm{p}, \mathrm{q})$ is the unit vector between pixel $\mathrm{p}$ and $\mathrm{q}$.

2.1.2 Optimal Path: Strong edge has low cost function value, and it can be regarded as searching for a path from start pixel to goal pixel with a minimal cumulative cost value. In order to avoid the effect of noise, increase the searching speed and further promote the image segmentation precision, many algorithms such as particle swarm optimization (PSO), pulse coupled neural network (PCNN) have been proposed in this domain. In Ref. 
[12], Guo optimized the live wire of shortest path algorithm with PCNN and got a better result than before. Then, Yu used PCNN to seek for optimal path which represented the object edge, and also obtained a desired segmentation effect [13]. Besides, Yi also achieved a better effect in segmentation precision through improving live wire segmentation with PSO [14].

Based on the research of visual cortex neuron of mammals, Eckhorn firstly proposed pulse coupled neural network [15]. In this study, live wire segmentation based on PCNN was adopted to capture trees in forestry image.

Applied in live wire segmentation of shortest path searching, Guo improved the math mode of PCNN [16], which is shown in formula 4.

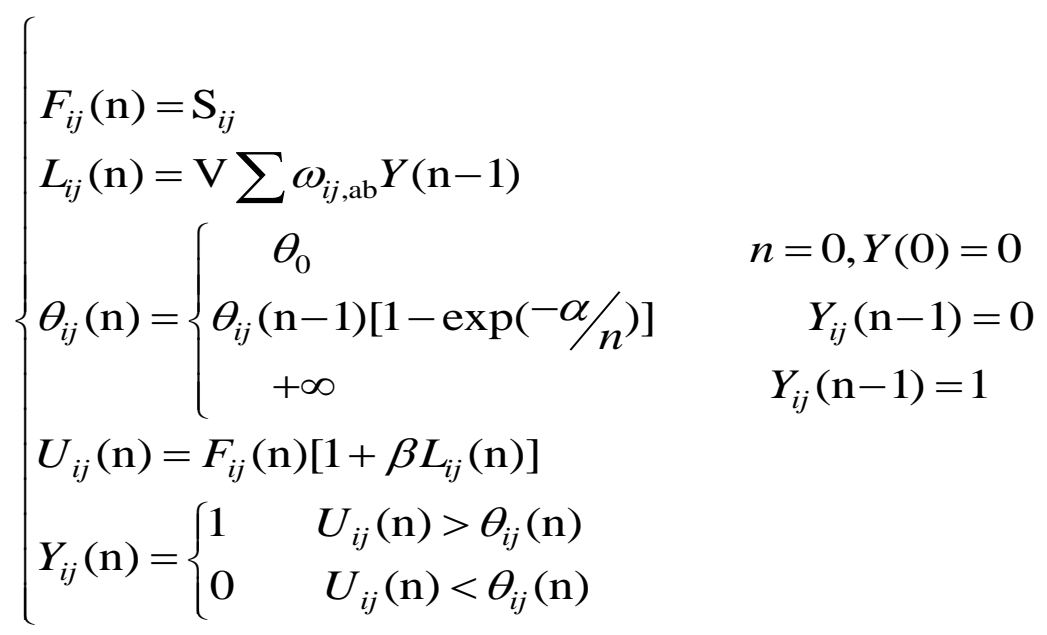

PCNN is a partially linked network (one layer and two dimensions). Each pixel can be regarded as the input of a neuron, and the neuron is connected with eight adjacent neurons.

In this mode, two features contribute to the probability in shortest path searching of live wire algorithm. One is the uniqueness of fire in each neuron, and the other is that the neuron can only be affected by the firstly received signal. In this theory, $\omega$, the synaptic gain strength between neurons is linearly correlated with its cost function. What is more, according to formula 4 , the duration that a signal transmits between neighbor neurons is $t+\omega$, where $t$ is the time spent on iteration. Thus, the smaller the cost function value is, the faster the signal transmits. This is the foundation of using PCNN in live wire segmentation method.

When the mode start, the start node produces pulse signal, and then the neuron with minimal cost function value will firstly receive the signal and output its pulse signal. As shown in formula 4 , its dynamic threshold, $\theta$, increases so rapidly that it will not output pulse signal any more. Thus we can trace the route of pulse signal from start node to start node, and capture the boundary of this object. This does help to promote segmentation rate and avoid the interference of noise.

\subsection{Image Enhancement}

Although improved by PCNN, the foundation of segmentation still lays on the pixel level of images, and the interference of noise and fake contour also exist. Therefore, image enhancement is needed to get high quality image. And then we take Hessian matrix to enhance image. The matrix of each pixel is the result of second order Gaussian partial derivative [17], which is:

$$
H=\left|\begin{array}{ll}
f_{x x} & f_{x y} \\
f_{y x} & f_{y y}
\end{array}\right|
$$


Here, $f$ is the convolution of the image with the scaled second order Gaussian partial derivative, and

$$
f_{x y}=\operatorname{Im} * \frac{\partial^{2}}{\partial x \partial y} G_{2 D}(\bar{x}, \sigma)
$$

where $G_{2 D}(\bar{x}, \sigma)$ is the Gaussian function:

$$
G_{2 D}(\bar{x}, \sigma)=\frac{1}{2 \pi \sigma^{2}} e^{-\frac{|\bar{x}|^{2}}{2 \sigma^{2}}}
$$

Based on the research of Frangi [18], we construct the filter with the eigenvalue of Hessian matrix, which denotes the curvature at the pixel $(x, y)$. Supposing $\lambda_{1}$ and $\lambda_{2}$ represent the absolute value of eigenvalues of Hessian matrix and $\lambda_{2}$ is the larger, then the filter we construct is:

$$
\text { output }=\exp \left(-\frac{\left[\frac{\lambda_{2}}{\lambda_{1}}\right]^{2}}{\beta}\right) *\left(1-\exp \left(-\frac{\left(\lambda_{1}\right)^{2}+\left(\lambda_{2}\right)^{2}}{\alpha}\right)\right)
$$

where $\alpha$ and $\beta$ are regulation parameters.

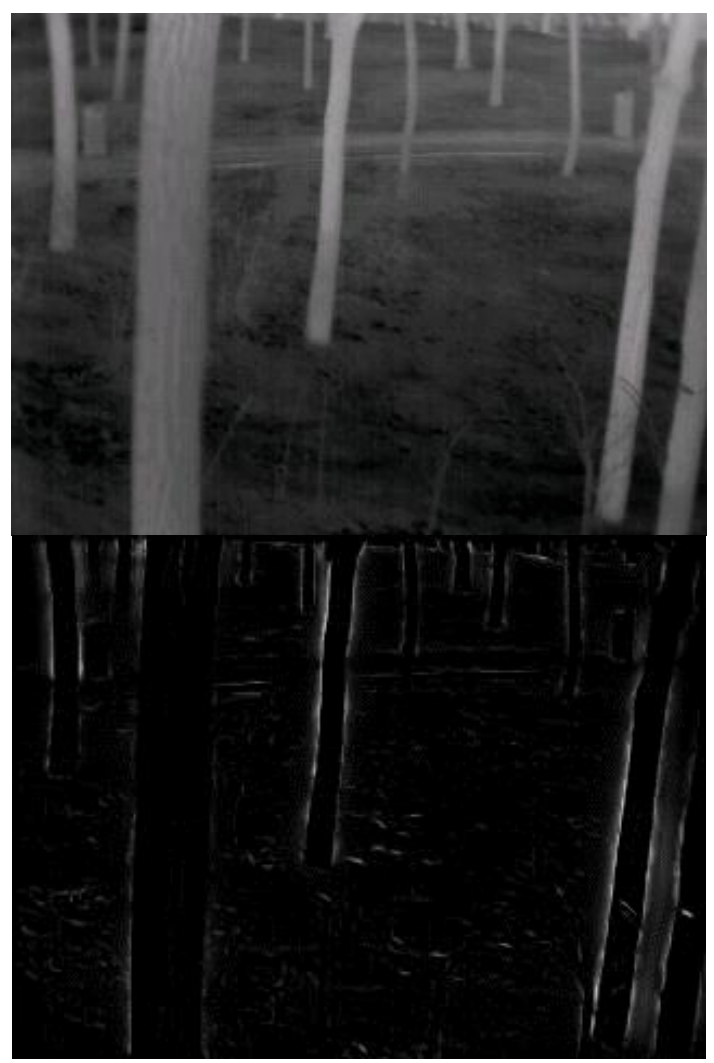

(a) Original image

(b) Enhanced image

Figure 2. Results after Image Enhancement 


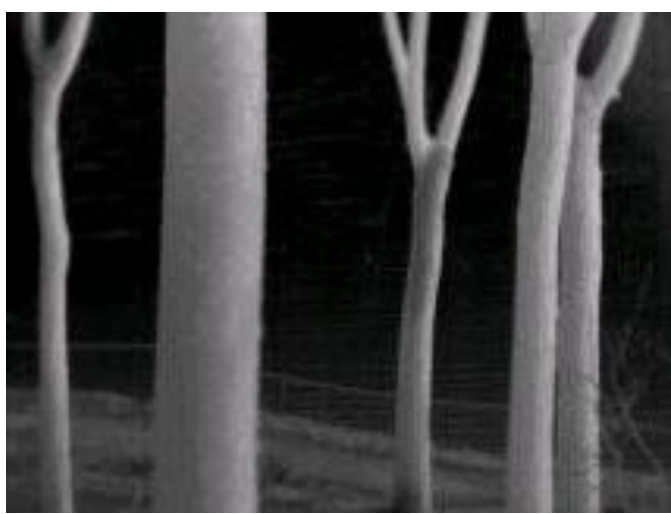

(a) Original image

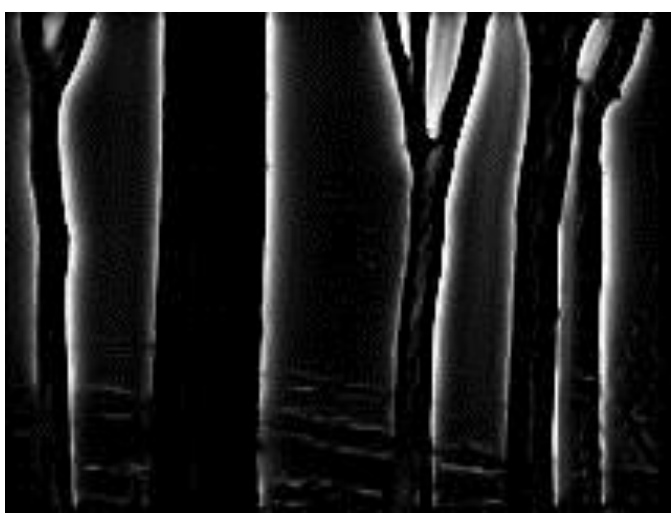

(b) Enhanced image

Figure 3. Results after Image Enhancement

Figure 2 and figure 3 present the original images and enhanced images. Compared with original image, the edge of trees in enhanced images is obviously enhanced, and the interference of the background is filtered largely. Besides, texture on trees in figure 2(a), which has an impact on segmentation effect, is filtered in figure 2(b). What's more, the grassland in original images is another element which affects trees' contour detection. And in figure 2(b) and figure 3(b) the land is largely weaken. With this enhanced image, we can obtain $f_{\text {canny }}(q)$ and $f_{\text {Log }}(q)$ precisely, and then the cost function becomes more reasonable in describing the contour of trees. Compared with original images, more edge information can be provided without interference.

\section{Result and Discussion}

\subsection{Image Capture}

Images were collected by Fluke ti55 infrared thermal camera in the National Olympic Forest Park, Beijing. Although it captured the information about temperature to avoid interference caused by visual light, the trees in these images were still difficult to be recognized because of the complicated surroundings.

\subsection{Segmentation Results}

Using live wire algorithm based on Hessian matrix and PCNN, we extracted the boundaries of trees in infrared thermal images. Since live wire algorithm was an interactive segmentation method, we should provide the start pixel and goal pixel according to visual sight.

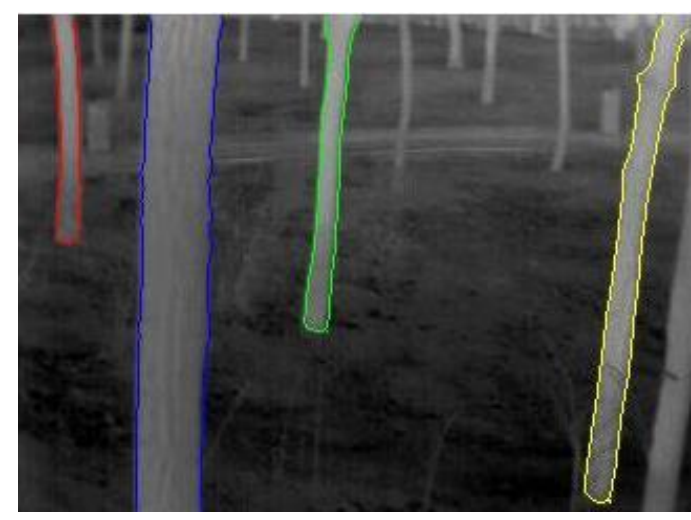

Figure 4. Segmentation Result 


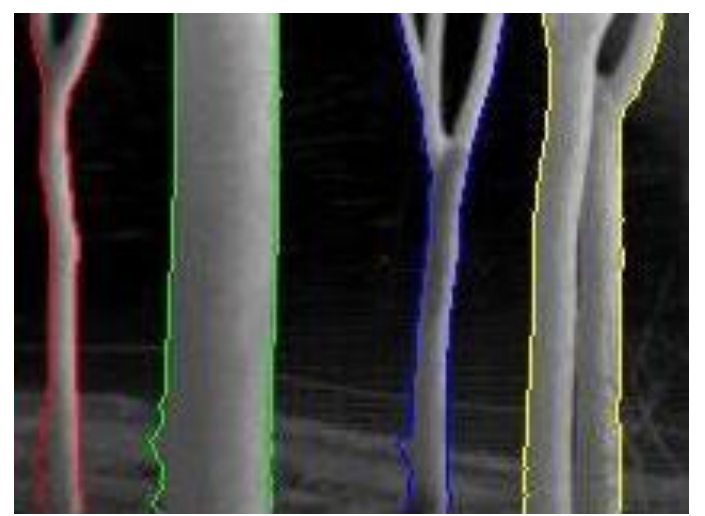

Figure 5. Segmentation Result

Figure 4 and figure 5 show the segmentation result of figure 2(a) and figure 3(a). Obviously, it is not difficult to get the trees visually in figure 2 (a) and figure 3(a). However, it is beyond our reach to obtain target information in the images for subsequent procedure in computer vision processing. Then, as shown in figure 4 and figure 5 , with the proposed method, the contour area can be extracted even at the position where confusion easily occurs. What is more, the data needed in intellectual identification can be obtained. On this basis, we can extract target information. Therefore, it becomes available to analyze some details of the target and its surroundings, including diameter, height and temperature, which is the foundation of target identification and intelligent monitoring. In addition, it can provide information for the harvester to decide fellable targets.

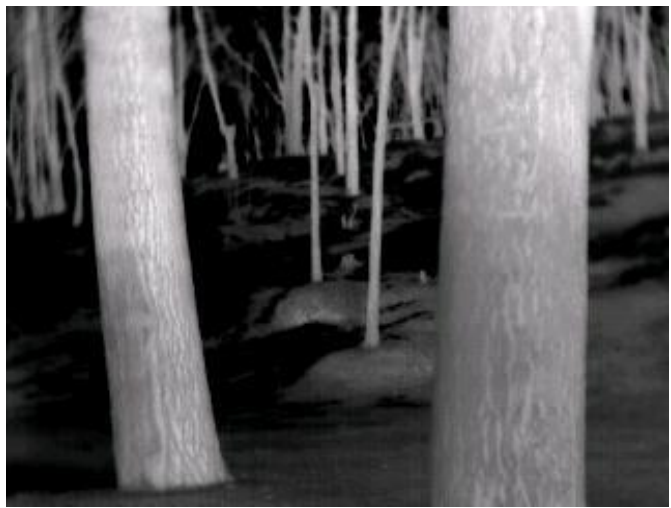

(a) Original image

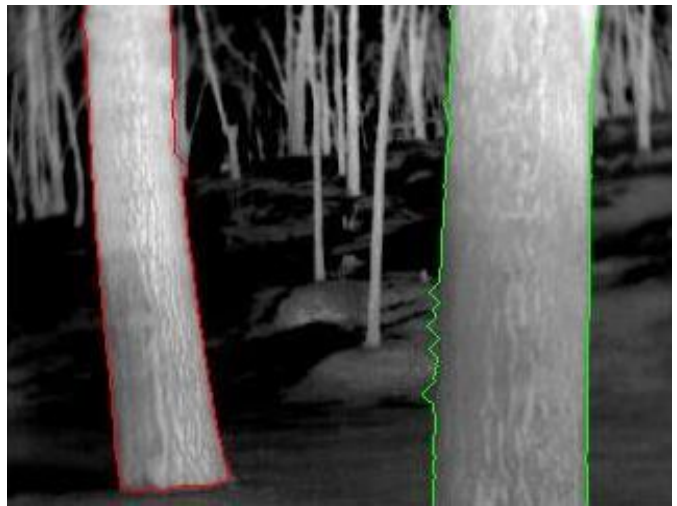

(b) Contour of trees

Figure 6. Extraction of Trees in Images

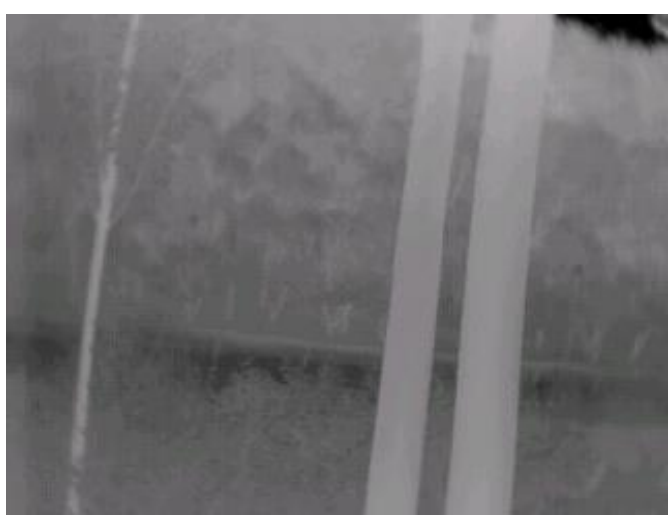

(a) Original image

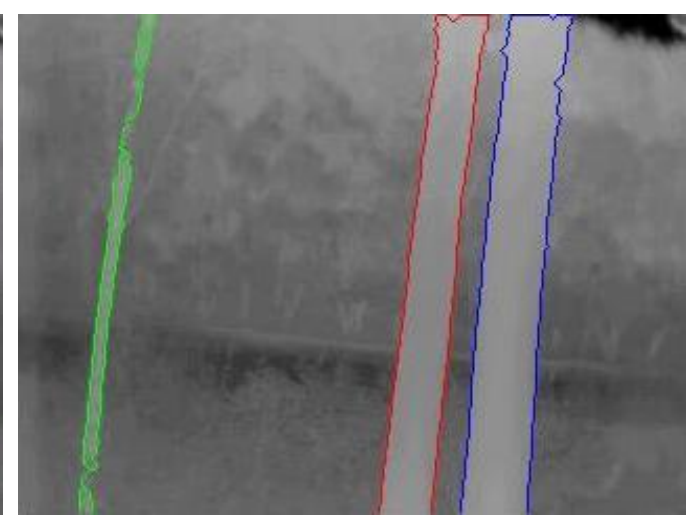

(b) Contour of trees

Figure 7. Extraction of Trees in Images 
The proposed method is feasible in capturing contour of trees. However, as shown in figure 6 and figure 7, segmentation result in seriously confused area might still be misled by surroundings, though enhanced before target extraction. In figure 6 , the contour of trees is precisely captured where no confusion occur. Nonetheless, affected by the confused background, error occurs on the contour of the right target. As for the left target in figure 7, the edge of the left tree could not be precisely obtained since it is sheltered seriously by plants. The mentioned situation is caused by the foundation of this method (pixel value) and has become the most powerful restraint in this domain. Thus, the utilization of proposed method is limited.

\section{Conclusions}

In this paper, a novel PCNN and Hessian based live wire segmentation was proposed to extract trees in forestry images, in which Hessian matrix was used to enhance image so as to avoid noise and confusion, and PCNN was to search for an optimal path in order to promote segmentation rate and precision. The result indicated that trees in image could be precisely extracted without interfered by the background, and then detail data including height, diameter and temperature could be obtained. Therefore, the proposed method would provide information for identification and monitoring, and promote the automatic and intelligent level in forestry production and management. However, in the situation of tiny difference on pixel value between target and background or sheltered by surroundings, the contour of trees could not be captured accurately. Therefore, further research is needed to promote the segmentation precision and broaden utilization range.

\section{Acknowledgement}

This paper is financially supported by Beijing Higher Education Young Elite Teacher Project (YETP0759), China Postdoctoral Science Special Foundation (2013T60070), 948 project supported by State Forestry Administration, China (Grant No. 2011-4-02).

\section{References}

[1] E F J Ring and K Ammer, "Infrared thermal imaging in medicine", Physiological measurement, vol. 33, no. 3, (2012), pp. 33-46.

[2] M. Kontos, R. Wilson and I. Fentiman, "Digital infrared thermal imaging (DITI) of breast lesions: sensitivity and specificity of detection of primary breast cancers", Clinical Radiology, vol. 66, no. 6, (2011), pp. 536-539.

[3] S. Zia, G. Romano, W. Spreer and C. Sanchez, "Infrared Thermal Imaging as a Rapid Tool for Identifying Water-Stress Tolerant Maize Genotypes of Different Phenology", Journal of Agronomy and Crop Science, vol. 199, no. 2, (2013), pp. 75-84.

[4] L. Yan, Z. Yu, N. Han and J. Liu, "Improved Image Fusion Algorithm for Detecting Obstacles in Forests", Journal of Digital Information Management, vol. 11, no. 5, (2013), pp. 378-384.

[5] X. Miu, Z. Dai and J. Chen, "Automatic Extraction of Coronary Vessel Based on Fuzzy C-Means Cluster", Proceedings of the 16th Chinese academy of electronic information theory academic annual meeting, (2009), part 1, pp. 243-248.

[6] J. Geng, "Research of Human Slice Color Image Segmentation", Nanjing University of Science and Technology, (2011).

[7] K. Thapaliyaa, J.-Y. Pyuna, C.-S. Parkb and G.-R. Kwona, "Level set method with automatic selective local statistics for brain tumor segmentation in MR images", Computerized Medical Imaging and Graphics, vol. 37, (2013), pp. 522-537.

[8] R. Sammouda, N. Adgaba, A. Touir and A. Al-Ghamdi, "Agriculture satellite image segmentation using a modified artificial Hopfield neural network", Computers in Human Behavior, vol. 30, (2014), pp. 436-441.

[9] A. Chodorowskia, U. Mattssonb, M. Langillec and G. Hamarneh, "Color Lesion Boundary Detection Using Live Wire", Medical Imaging 2005: Image Processing, vol. 5747, (2005), pp. 1589-1595.

[10] E N. Mortensen, B S. Morse, W A Barrett and J K Udupa, "Adaptive Boundary Detection Using 'Live Wire' Two-Dimensional Dynamic Programming", IEEE proceedings of Computers in Cardiology (1992), Durham, USA, pp. 635-638.

[11] G. Li, "Study and Application of Interactive Image Segmentation", Northeastern University, (2008). 
[12] G. Li, L. Gao, J. Wu, K. Lu and K. Wang, "New Algorithm of Live Wire Based on PCNN Simulation Research", Journal of System Simulation, vol. 20, no. 14, (2008), pp. 3707-3711.

[13] H. Yu, "Research of Interactive Image Segmentation", Northeastern University, (2010).

[14] Y. Yi, L. Gao, Z. Cui and S. Shi, "PSO-Based Live Wire Interactive Image Segmentation Algorithm", Journal of Northeastern University( Natural Science)", vol. 32, no. 2,( 2011), pp. 193-201.

[15] T. Lindblad and J.M Kinser, Image Processing Using Pulse-Coupled Neural Networks, Higher Education Press, China, (2008).

[16] G. Li and K. Wang, "A New Image Segmentation on Live wire based on MPCNN", International Conference on Services Science, Management and Engineering, (2010), pp. 306-309.

[17] X. Jiang, J. Xiong, Y. Qiu and D. Fan, "Enhancement Filter Algorithm of Retina Blood Vessels Based on Hessian Matrix Features", Journal of East China Jiaotong University, vol. 30, no. 3, (2013), pp. 37-43.

[18] A. F. Frangi, W. J. Niessen and K. L. Vincken, "Multi-scale vessel enhancement filtering", Medical Image Computing and Computer-Assisted Intervention, LNCS 1496, Berlin: Springer, (1998), pp. 130-137.

\section{Authors}

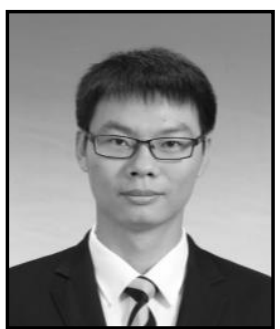

Zheng Yu, He was born in China, and received his bachelor degree in Automation from Beijing Forestry University in 2010. Now he is pursuing his master degree in Control theory and control engineering from Beijing Forestry University. His research interests concentrate on image processing and image fusion. 